\title{
Personality Beliefs in Obsessive-Compulsive Disorder: How Are They Related to Symptom Severity?
}

\author{
Aysegul Kart ${ }^{1}$ and Bengu Yucens ${ }^{2} \bowtie$ \\ ${ }^{1}$ Department of Psychiatry, Bakirkoy Mental and Nervous Diseases Training and Research Hospital, Istanbul, Turkey \\ ${ }^{2}$ Department of Psychiatry, Pamukkale University Faculty of Medicine, Denizli, Turkey
}

Objective The comorbidity of obsessive-compulsive disorder (OCD) and personality disorders (PDs) is frequent but there are conflicting findings about which PDs are the most common. This study aimed to investigate the personality beliefs that exist on a more pathological level among OCD patients, to explore the association between personality beliefs and OCD severity, and to clarify the mediator effect of depression in this relationship.

Methods 202 OCD patients and 76 healthy controls with similar sociodemographic features were included in the study. The Personality Belief Questionnaire-Short Form was administered to both groups. The Yale-Brown Obsessions and Compulsions Scale, Beck Depression Inventory, and the Beck Anxiety Inventory were administered only to the clinical sample.

Results The dependent, histrionic, paranoid, borderline, and avoidant personality subscale scores were significantly higher in the OCD group than in the control group. There was an association only between OCD severity and narcissistic personality beliefs, also depression mediated the relationship between narcissistic personality and OCD severity.

Conclusion Some personality beliefs at a pathological level are more common among OCD patients. Personality beliefs, as well as depression, should be routinely assessed, as they may affect OCD severity, help-seeking behavior, and response to treatment.

Psychiatry Investig 2020;17(8):822-828

Key Words Obsessive-compulsive disorder, Personality, Depression, Cognitive behavioral therapy.

\section{INTRODUCTION}

Obsessive-compulsive disorder (OCD) is a chronic, disabling condition characterized by distressing recurring and persistent thoughts, and/or by the need to perform actions in order to reduce the feeling of distress. ${ }^{1}$ In studies that focus on the cognitive structures in OCD, non-functional beliefs and appraisals as the exaggerated perception of responsibility, maladaptive interpretation of the personal significance of thoughts, perfectionism, and intolerance of uncertainty have been addressed. ${ }^{2}$ Of the studies conducted to date, the predominant findings showed that when compared to non-clinical samples, those with OCD have a higher prevalence of comorbid per-

\footnotetext{
Received: March 27, 2020 Revised: May 5, 2020

Accepted: June 9, 2020

$\bowtie$ Correspondence: Bengu Yucens, MD

Department of Psychiatry, Pamukkale University Faculty of Medicine, Denizli, PA:20100, Turkey

Tel: +90 505263 3138, E-mail: dr.bengubaz@yahoo.com

(a) This is an Open Access article distributed under the terms of the Creative Commons Attribution Non-Commercial License (https://creativecommons.org/licenses/bync/4.0) which permits unrestricted non-commercial use, distribution, and reproduction in any medium, provided the original work is properly cited.
}

sonality disorders (PDs) that ranged from 9 to $88 \%{ }^{3}$ The cluster $\mathrm{C}$ comorbid PDs have been found to occur most frequently, while cluster A and B PDs such as narcissistic, schizoid and antisocial PDs are rarely diagnosed in OCD. ${ }^{4,5}$ Additionally, the obsessive-compulsive PD (OCPD) that seemed to be most frequent in the studies typically focus on a single PD. ${ }^{6,7}$

According to cognitive theory, the basis of a PD is revealed in the dysfunctional beliefs that characterize and perpetuate it. Dysfunctional beliefs are a primary focus of treatment in cognitive therapy of PDs. If dysfunctional beliefs are identified correctly, these beliefs reflect cognitive themes indicating the developmental history of the patient, compensatory strategies, and dysfunctional reactions toward existing conditions. ${ }^{8}$ Inquiring about personality can help to clarify whether these beliefs may play an important role in treatment plan and response, particularly in cognitive behavioral therapy (CBT) of OCD. ${ }^{8}$

The association between the severity of OCD symptoms and comorbid PDs has been revealed in previous studies. Besides the conflicting findings of this relationship, there has been no sufficient study focusing on the common dysfunc- 
tional beliefs in OCD and PDs. Bulli et al. ${ }^{3}$ found that relative risk of having a comorbid PD was 12 times as high in those with moderate symptom severity and 39 times as high in those with high symptom severity in patients with OCD. In contrast, some studies suggest that OCD patients with comorbid PDs do not have more severe OCD symptoms. ${ }^{9}$ Whether or not comorbid PDs affect symptom severity, these patients need more comprehensive treatment rather than a treatment focused solely on symptoms, as their problemsolving skills are lower and this comorbidity has a potential impact on treatment compliance and response. ${ }^{10}$ The role of depressive symptoms should also not be overlooked when evaluating the relationship between personality and OCD severity. Comorbidity is the rule rather than the exception, with major depressive disorder (MDD) being one of the most frequent comorbid diagnoses in OCD. ${ }^{11,12}$ Nearly $50 \%$ of OCD patients present with an additional diagnosis of MDD and symptom severity of these patients has been found to be higher than in those without the additional diagnosis. ${ }^{13}$

Instead of variable prevalence rates of PDs, it may be more useful for planning the CBT of OCD to investigate common dysfunctional personality beliefs. As therapist and patient work together to identify and modify these key beliefs, improvements may be seen across many areas of functioning. Besides, the evaluation of the beliefs would be also commensurate for the trait-based model of personality proposed in The Diagnostic and Statistical Manual of Mental Disorders- 5 (DSM-5). ${ }^{1}$ According to the hybrid model of "Alternative DSM-5 Model for Personality Disorders" for the assessment and diagnosis of personality pathology are dimensional ratings of 1) the severity of impairment in personality (self and interpersonal) functioning and 2) 25 pathological personality trait "facets" organized into five broad trait "domains." In line with this information, identification of the pathological level of personality beliefs instead of personality disorders may help to integrate pathological personality and normative personality into a single coherent model.

Few studies have attempted to clarify or characterize the personality beliefs and its clinical correlates in OCD. In this study, we hypothesized that some personality beliefs reach more pathological levels in patients with OCD than in healthy controls and are associated with the severity of OCD. However, it is possible that the findings pertaining to personality beliefs in pathological levels can be explained by vulnerability to depressive symptoms, so controlling for depression could help to elucidate the unique relationship between personality beliefs and the severity of OCD.

\section{METHODS}

\section{Participants and procedure}

A total of 202 patients with OCD who were admitted to the Department of Psychiatry at Bakirkoy Mental and Nervous Diseases Training and Research Hospital between March 2018 and December 2018 were included in this study. Diagnostic assessments were conducted by psychiatrists. The inclusion criteria were a diagnosis of primary OCD according to DSM- 5 criteria, age between 18 and 65 years, being able to read and fill out forms, and voluntary consent. Exclusion criteria were diagnosis of comorbid neurocognitive, psychotic or bipolar disorders. Additionally, 76 healthy controls with similar sociodemographic features participated in the study. A face-to-face interview was conducted with all patients and healthy volunteers. The procedures involved in this research protocol were fully explained to patients, who signed informed consent before being included in the study. The protocol was approved by the Local Ethics Committee, the protocol date and number was 06.02.2018-127.

\section{Measures}

The Sociodemographic Data Form and the Personality Belief Questionnaire-Short Form (PBQ-SF) were administered to all participants. Yale-Brown Obsessions and Compulsions Scale (Y-BOCS), Beck Depression Inventory (BDI), Beck Anxiety Inventory (BAI) was administered only to the patients with OCD.

\section{Sociodemographic data form}

A structured questionnaire was used to gather information about demographic features including age, sex, marital status, and educational level.

\section{Personality Belief Questionnaire-Short Form (PBQ-SF)}

Beck and Beck ${ }^{14}$ developed the Personality Belief Questionnaire $(\mathrm{PBQ})$ based on cognitive theory and clinical observations for Axis-II disorders. The PBQ has been used to measure cognitive mechanisms of treatment outcomes, and has been found to be sensitive to changes in beliefs. The validity and reliability of the Turkish version of the original PBQ was evaluated by Turkcapar et al..$^{15} \mathrm{~A}$ cut-off score was not defined in this study, thus a cut-off score was not used in the current study. The researchers that developed the original version of this form subsequently developed a PBQ-SF containing 65 distinctive items selected from the original PBQ form. ${ }^{16}$ The validity and reliability of the Turkish version of the original PBQ-SF was evaluated by Taymur et al. ${ }^{17}$ In total, 10 scales assess 10 personality disorders: paranoid, schizoid, antisocial, borderline, histri- 
onic, narcissistic, avoidant, dependent, obsessive-compulsive, and passive-aggressive. PBQ-SF is a self-report Likert-type questionnaire that is scored from 0 ("I don't believe it at all.") to 4 ("I believe it totally."). Example items from the PBQ-SF include "I am not influenced by others in what I decide to do." (schizoid), "I can not tolerate unpleasant feelings." (avoidant), "If I am not loved, I will always be unhappy." (dependent), and "The only way I can preserve my self-respect is by asserting myself indirectly." (passive-aggressive).

\section{Yale-Brown Obsessions and Compulsions Scale (Y-BOCS)}

The severity and types of OCD symptoms were assessed using the Y-BOCS. ${ }^{18}$ The Y-BOCS is a clinician-rated scale, consisting of 10 items to assess the severity of obsessions and compulsions with respect to time spent, interference, distress, resistance, and control. Each item is rated by a clinician from 0 (no symptoms) to 4 (extreme symptoms) (total range $0-40$ ). The Turkish adaptation, validity, and reliability study was conducted by Tek et al. ${ }^{19}$

\section{Beck Depression Inventory (BDI)}

The BDI is a 21-item self-report inventory and it is one of the most widely used instruments for measuring the severity of depression and clinical improvements. Each item is scored on a scale from 1 to 3 , and total scores are calculated by summing the scores on all items. The Turkish-language validity and reliability test was conducted by Hisli. ${ }^{20}$

\section{The Beck Anxiety Inventory (BAI)}

BAI is a 21-item self-report scale describing subjective, somatic, or panic-related symptoms, each consisting of four statements about physiological and cognitive components of anxiety. ${ }^{21}$ Items are rated on a Likert-type scale from 0 to 3 , reflecting how much anxious have felt over the past week. Possible scores range from 0 to 63 .

\section{Statistical analysis}

IBM SPSS 15.0 (SPSS Inc., Chicago, IL, USA) was used for statistical analyses. Group differences in sociodemographic variables were computed through the chi-square test. Normal distribution of the data was evaluated with the KolmogorovSmirnov distribution test. The Independent Samples Test was used to compare the quantitative variables that were normally distributed. In all tables, the numerical variables are presented as mean \pm standard deviation (SD) and the categorical variables are presented as numbers of percentages (\%). Correlations between PBQ-SF subscale scores and Y-BOCS scores were assessed using Pearson correlations analysis. Significance levels were set at $\mathrm{p}<0.05$ and $\mathrm{p}<0.001$. Multiple linear regression analysis was used to determine whether personality beliefs predict the OCD severity and depression mediates the relationship between personality beliefs and OCD severity. Sobel test was used for mediation test. This test showed whether depression (mediator) carried the influence of personality beliefs to OCD severity.

\section{RESULTS}

Of these 202 OCD patients, 121 (59.9\%) were female and $81(40.1 \%)$ were male. The mean age was $31.22 \pm 9.49$ years. $149(73.8 \%)$ were receiving treatment and $42(20.7 \%)$ had been diagnosed with MDD according to DSM-5 criteria. In the sociodemographic features, there was no statistically significant difference in the sex distributions, mean age, marital status, the educational level between groups. The sociodemographic and clinical characteristics of the study sample are shown in Table 1.

The results showed that the OCD group obtained higher scores than the control group for the dependent, histrionic, paranoid, borderline, and avoidant personality subscale scores, whereas there was no significant difference between groups with respect to passive-aggressive, obsessive-compulsive, antisocial, narcissistic and schizoid personality subscale scores (Table 2).

The Y-BOCS mean score of OCD patients was 21.18 \pm 7.21 and was positively correlated with BDI $(r=0.269, \mathrm{p}<0.001)$, BAI ( $\mathrm{r}=0.174, \mathrm{p}=0.013)$, avoidant $(\mathrm{r}=0.224, \mathrm{p}=0.001)$, dependent $(r=0.235, p=0.001)$, passive-aggressive $(r=0.197, p=0.005)$, obsessive-compulsive $(\mathrm{r}=0.210, \mathrm{p}=0.003)$, antisocial $(\mathrm{r}=0.192$,

Table 1. Sociodemographic features of participants

\begin{tabular}{lrcc}
\hline & OCD & Healthy controls & p \\
& $\mathrm{N}=202$ & $\mathrm{~N}=76$ & \\
\hline Sex, N (\%) & & & $0.768^{*}$ \\
$\quad$ Male & $81(40.1)$ & $29(38.2)$ & \\
$\quad$ Female & $121(59.9)$ & $47(61.8)$ & \\
Marital status, N (\%) & & & $0.732^{*}$ \\
$\quad$ Married & $87(56.9)$ & $31(59.2)$ & \\
$\quad$ Single & $115(43.1)$ & $45(40.8)$ & \\
Education (year), N (\%) & & & $0.090^{*}$ \\
0-5 & $20(26.3)$ & $34(16.9)$ & \\
6-8 & $10(13.2)$ & $22(10.9)$ & \\
9-12 & $23(30.3)$ & $52(25.9)$ & \\
$\geq 13$ & $23(30.3)$ & $93(46.3)$ & \\
Age (mean \pm SD) & $31.22 \pm 9.49$ & $33.22 \pm 9.37$ & $0.118^{\dagger}$ \\
\hline
\end{tabular}

*chi-square test, ${ }^{\text {independent samples test, quantitive variables " } \mathrm{N}}$ (per cent)." SD: standard deviation, OCD: obsessive-compulsive disorder 
$\mathrm{p}=0.006)$, histrionic $(\mathrm{r}=0.168, \mathrm{p}=0.017)$, and narcissistic $(\mathrm{r}=0.226, \mathrm{p}=0.001)$, paranoid $(\mathrm{r}=0.139, \mathrm{p}=0.048)$ and borderline $(r=0.253, p<0.001)$ personality subscale scores; whereas the schizoid ( $\mathrm{p}=0.091)$ personality subscale score showed no significant correlation (not shown in the table).

The multiple hierarchical linear regression analysis was conducted to test whether personality beliefs predicted OCD symptom severity. As BDI and BAI total scores showed a statistically significant positive correlation with Y-BOCS total score, these variables were included in the first block with age and sex variables in order to control their effects, and personality beliefs subscale scores were included in the second block. The model obtained in linear regression analysis was statistically significant $[\mathrm{F}(5,200)=4.692, \mathrm{p}<0.001]$. The results demonstrate that when age, sex, BDI and BAI variables were controlled only the narcissistic personality beliefs predicted YBOCS total score statistically significant (Table 3 ). The mediator effect of BDI score was evaluated in the relationship between narcissistic personality beliefs and Y-BOCS total scores

Table 2. Comparison of personality beliefs subscale scores between OCD and healthy control groups

\begin{tabular}{|c|c|c|c|c|}
\hline & $\begin{array}{c}\text { OCD } \\
\mathrm{N}=202\end{array}$ & $\begin{array}{l}\text { Healthy controls } \\
\qquad \mathrm{N}=76\end{array}$ & \multirow[t]{2}{*}{$\mathrm{t}$} & \multirow[t]{2}{*}{$\mathrm{p}$} \\
\hline \multicolumn{3}{|c|}{ Mean \pm SD } & & \\
\hline Dependent & $11.65 \pm 7.22$ & $6.97 \pm 4.49$ & -6.468 & $<0.001^{*}$ \\
\hline Passive-agressive & $12.58 \pm 5.81$ & $11.42 \pm 5.44$ & -1.518 & 0.130 \\
\hline $\begin{array}{l}\text { Obsessive- } \\
\text { compulsive }\end{array}$ & $13.35 \pm 6.28$ & $12.82 \pm 5.68$ & -0.634 & 0.527 \\
\hline Antisocial & $9.50 \pm 5.81$ & $8.17 \pm 5.13$ & -1.765 & 0.079 \\
\hline Narcissistic & $9.22 \pm 5.77$ & $8.80 \pm 5.43$ & -0.549 & 0.583 \\
\hline Histrionic & $8.64 \pm 6.64$ & $6.73 \pm 5.36$ & -2.468 & $0.015^{*}$ \\
\hline Schizoid & $12.75 \pm 5.93$ & $12.53 \pm 6.31$ & -0.268 & 0.789 \\
\hline Paranoid & $12.25 \pm 6.51$ & $9.07 \pm 6.45$ & -3.630 & $<0.001^{*}$ \\
\hline Borderline & $12.35 \pm 6.67$ & $6.42 \pm 4.48$ & -8.522 & $<0.001^{*}$ \\
\hline Avoidant & $14.31 \pm 5.65$ & $11.92 \pm 5.29$ & -3.202 & $0.002^{*}$ \\
\hline
\end{tabular}

${ }^{*} \mathrm{p}<0.05$. SD: standard deviation, OCD: obsessive-compulsive disorder
(Figure 1). In order to test mediation, a number of assumptions need to be met. The independent, mediator and dependent variables must be significantly associated and the mediator must also be associated with dependent variables. In the first regression model, the effect of the narcissistic personality beliefs variable on Y-BOCS decreased with the addition of BDI to the regression analysis in the third model, but it remained a statistically significant predictor. An increase of 1 point in the BDI was observed to result in an increase of 0.14 points in the Y-BOCS total score, whereas a 1-point increase in the narcissistic personality beliefs scores increased the Y-BOCS total score by 0.19 point. In order to conclude that the BDI variable predicts the Y-BOCS variable as a partial mediator between narcissistic personality beliefs and Y-BOCS, it is necessary to look at the significance of $\mathrm{z}$ value calculated by the Sobel test. According to the Sobel test, $\mathrm{z}=2.644$ ( $\mathrm{p}=0.008)$. A statistically significant $\mathrm{z}$ value indicates that depression mediates the relationship between narcissistic personality beliefs factor and OCD symptom severity. The data of the coefficients and significance of the models are presented in Table 4.

\section{DISCUSSION}

OCD patients exhibited dependent, histrionic, paranoid, borderline, and avoidant personality beliefs at a more patho-

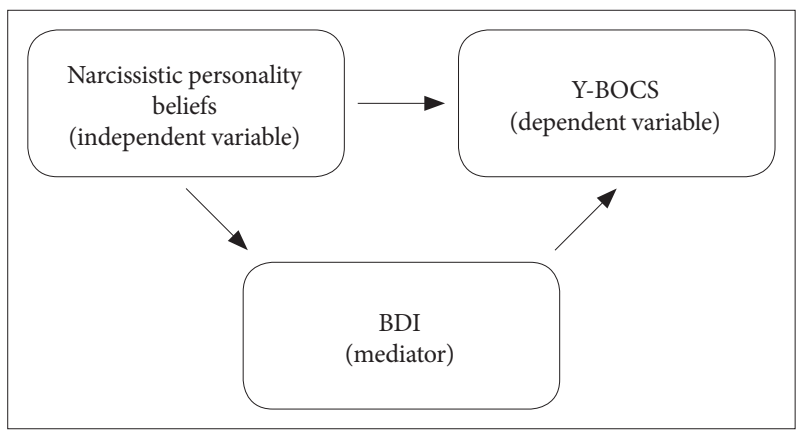

Figure 1. The mediator effect of $\mathrm{BDI}$ scores in the relationship between narcissistic personality beliefs and Y-BOCS total scores. BDI: Beck Depression Inventory, Y-BOCS: Yale-Brown Obsessions and Compulsions Scale.

Table 3. Multiple hierarchical linear regression analysis results associated with the Y-BOCS scores

\begin{tabular}{lccccc}
\hline & B (LBCI-UBCI) & SE & Beta & t & Significance \\
\hline Constant & $13.576(8.763-18.389)$ & 2.440 & & 5.563 & 1.578 \\
Age & $0.082(-0.021-0.185)$ & 0.052 & 0.108 & 0.001 & 0.116 \\
Sex & $0.328(-1.648-2.304)$ & 1.002 & 0.022 & 0.328 & 0.744 \\
BDI & $0.138(0.027-0.249)$ & 0.056 & 0.214 & 2.446 & $0.015^{*}$ \\
BAI & $0.005(-0.091-0.100)$ & 0.048 & 0.008 & 0.093 & 0.926 \\
Narcissistic personality & $0.187(0.011-0.364)$ & 0.090 & 0.150 & 2.090 & $0.038^{*}$ \\
\hline
\end{tabular}

${ }^{*} \mathrm{p}<0.05$. Dependent variable: Y-BOCS, independent variables: age, sex, BDI and BAI scores, dependent, passive-aggressive, obsessive- compulsive, antisocial, narcissistic, histrionic, schizoid, paranoid, borderline, and avoidant personality subscale scores. SE: standard error, BDI: Beck Depression Inventory, BAI: Beck Anxiety Inventory, Y-BOCS: Yale-Brown Obsessions and Compulsions Scale 
Table 4. Linear regression analysis results associated with the mediator effect of depression on OCD severity

\begin{tabular}{lcccccr}
\hline \multicolumn{1}{c}{ Predictor variables } & $\begin{array}{c}\text { Predicted variable } \\
\text { (dependent variable) }\end{array}$ & $\mathrm{R}^{2}$ & $\mathrm{~F}$ & $\mathrm{~B}$ & $\mathrm{SE}$ & $\mathrm{p}$ \\
\hline Narcissistic personality & Y-BOCS & 0.051 & 11.296 & 0.283 & 0.084 & 0.001 \\
Narcissistic personality & BDI & 0.093 & 22.154 & 0.592 & 0.126 & $<0.001$ \\
BDI & Y-BOCS & 0.095 & 11.434 & 0.143 & 0.043 & 0.001 \\
Narcissistic personality & & & & 0.199 & 0.086 & 0.021 \\
\hline
\end{tabular}

Sobel $\mathrm{Z}=2.654, \mathrm{p}=0.008$

SE: standard error, BDI: Beck Depression Inventory, Y-BOCS: Yale-Brown Obsessions and Compulsions Scale

logical level when compared with healthy controls. However, as depression is highly comorbid with OCD and because previous studies had found an influence of depression on observed personality outcomes, ${ }^{22}$ the relationship between personality beliefs, depression and OCD was explored in the current study. The results showed that the narcissistic personality beliefs only predicted the symptom severity in OCD patients, and depression had a mediator effect in the relationship.

Although it is generally accepted that PDs are more common in OCD patients, there are different findings in the literature about which PDs are more common. The most clinically relevant co-occurring PD in OCD is OCPD, followed by schizotypal PD and borderline PD. ${ }^{23}$ Pena-Garijo et al. ${ }^{4}$ found higher prevalence rate of PDs in $41 \mathrm{OCD}$ patients with regard to the general population with Structured Clinical Interview for DSM-IV Axis II Personality Disorders (SCID-II) and the most prevalent were respectively, obsessive-compulsive, avoidant and dependent PDs. In the same study, as a dimensional instrument, International Personality Disorder Examination (IPDE) scores of the OCD group showed significantly higher scores when compared to control group in all PDs except paranoid and histrionic PDs. ${ }^{4}$ In another study with 108 OCD patients, the prevalence of PDs, as determined by the SCID-II questionnaire was $74 \%$ and all categories, except histrionic PD, were significantly more prevalent in the OCD group compared to the nonneurotic control group. ${ }^{24}$ In this study, both some results were found that were compatible with the literature and some found that were incompatible. Dependent and avoidant personality belief levels in cluster $\mathrm{C}$ PDs were higher than those of healthy subjects, however, obsessive-compulsive personality belief levels were similar between the two groups. The fact that both OCD and cluster C PDs are characterized by certain behaviors that are maladaptive responses to cope with anxiety may explain this relationship. Friborg et al. ${ }^{5}$ did not have a noticeably higher occurrence of a comorbid OCPD compared with the other cluster C PDs. While the higher level of borderline personality beliefs is consistent with the literature, higher levels of paranoid and histrionic personality beliefs are other than expected. The variable results for comorbid PD types are probably due to several methodological differences including the sample selection, instruments for PDs assessment, research designs, focus on specific PDs rather than the number of PDs.

Melca et al. ${ }^{25}$ suggested that patients with OCD and comorbid PDs tend to exhibit more severe symptoms. In a study with 159 OCD patients Cluster B was found to be the most important cluster affecting Y-BOCS total score. ${ }^{3}$ Besides in a longterm longitudinal study, the patients with borderline PD were found to be more likely to relapse after having reached a remission status at 7-year follow-up. ${ }^{26}$ One systematic review showed that poorer treatment outcome in OCD patients was associated only with the presence of any DSM Cluster A personality disorder, as well as the presence of narcissistic personality disorder or at least two personality disorders, whereas DSM Cluster B or Cluster $\mathrm{C}$ personality disorders were not consistently associated with treatment outcomes. ${ }^{27}$ Thus, only the narcissistic personality beliefs level was found to be associated with OCD severity in the current study. If the relationship between personality and OCD severity is to be determined clearly, factors such as anxiety and depression should also be taken into consideration. Because MDD is a common co-occurring diagnosis as found $28.4 \%$ in a survey of OCD comorbidity in more than 3,700 participants across seven countries represents the largest international evaluation of OCD comorbidity in the literature thus $\mathrm{far}^{28}$ also $20.7 \%$ in the current study. Thus, Rees et al. ${ }^{22}$ suggested that depression would mediate the relationship between personality traits and OCD symptom severity. In this study mediator effect of depression was also investigated, with findings similar to those in the literature. A treatment study that investigated the mediators of change in behavioral versus cognitive therapy in OCD patients found that the improvements in OCD symptoms were mediated by reductions in depressed mood in both behavioral and cognitive therapy. ${ }^{29}$

CBT is the empirically supported treatment option for OCD and there are studies investigating the role of comorbid PDs on the treatment outcomes. Fricke et al. ${ }^{9}$ showed that OCD patients with and without comorbid PDs benefited equally from a multimodal tailored CBT intervention involving ERP, cognitive restructuring and a stress-management training. In a study 
evaluating the impact of comorbid PDs on the treatment outcome, the Exposure with Response Prevention (ERP) treatment was found to be effective and comorbid PDs were not a significant predictor of treatment failure. ${ }^{30}$ These results may be attributed to the effects of CBT on dysfunctional beliefs. Such a treatment could attenuate the negative effects of schemas, thus enhancing the treatment progress. Consequently, it is important to investigate the personality beliefs in pathologic level; thus, these beliefs may be a marker of severe symptomatology in OCD and play an important role in the CBT process.

There are some limitations that should be mentioned in this study. Firstly, the use of a self-rated scale such as PBQ-SF instead of a structured clinical interview of PDs may be seen as a limitation. Self-reports like PBQ-SF are often affected by a tendency for patients to self-identify more pathology than clinicians would do through interviews; however, this possibility is also valid for the control group, and we therefore consider that it will not affect the results. Additionally, this dimensional assessment may provide more detailed information about the cognitive structure; this diversity is considered to be the distinguishing feature of the study. Secondly, although linear regression analysis showed a relationship between the personality beliefs and symptom severity variables, this study was based on a cross-sectional design, and as such alternative temporal orders have not been ruled out; it is also possible that these relationships are bi-directional. In fact, personality features are thought to be lifelong, beginning in childhood, and OCD develops later. Also, the sampling method did not facilitate the collection of information about the age of onset and treatment factors, so they were not controlled. Thus, it is possible that treatment history affected the results of the regression analysis.

In conclusion, clinicians should be aware that OCD and dysfunctional personality beliefs in a pathological level are frequently coexistent, and that this association often involves more than one personality type. CBT may be used as an efficacious treatment option in OCD patients with pathological levels of comorbid personality beliefs. The identification early in therapy of the key beliefs can help the therapist focus treatment more efficiently.

\section{Acknowledgments}

None.

\section{Conflicts of Interest}

The authors have no potential conflicts of interest to disclose.

\section{Author Contributions}

Conceptualization: Aysegul Kart, Bengu Yucens. Data curation: Aysegul Kart, Bengu Yucens. Formal analysis: Aysegul Kart, Bengu Yucens. Investigation: Aysegul Kart, Bengu Yucens. Methodology: Aysegul Kart, Bengu Yucens. Project administration: Aysegul Kart, Bengu Yucens. Resources:
Aysegul Kart, Bengu Yucens. Software: Aysegul Kart, Bengu Yucens. Supervision: Aysegul Kart, Bengu Yucens. Validation: Aysegul Kart, Bengu Yucens. Visualization: Aysegul Kart, Bengu Yucens. Writing-original draft: Aysegul Kart, Bengu Yucens. Writing_review \& editing: Aysegul Kart, Bengu Yucens

\section{ORCID iDs}

Bengu Yucens https://orcid.org/0000-0002-4721-7288

Aysegul Kart https://orcid.org/0000-0003-1248-835X

\section{REFERENCES}

1. American Psychiatric Association. Diagnostic and Statistical Manual of Mental Disorders, 5th Edition (DSM-5). Washington DC: American Psychiatric Publishing; 2013.

2. Salkovskis PM, Wroe AL, Gledhill A, Morrison N, Forrester E, Richards $\mathrm{C}$, et al. Responsibility attitudes and interpretations are characteristic of obsessive compulsive disorder. Behav Res Ther 2000;38:347-372.

3. Bulli F, Melli G, Cavalletti V, Stopani E, Carraresi C. Comorbid personality disorders in obsessive-compulsive disorder and its symptom dimensions. Psychiatr Q 2016;87:365-376.

4. Pena-Garijo J, Edo Villamón S, Meliá de Alba A, Ruipérez MÁ. Personality disorders in obsessive-compulsive disorder: a comparative study versus other anxiety disorders. Scientific World Journal [Internet]. 2013. Available at: https://doi.org/10.1155/2013/856846. Accessed May 10, 2019.

5. Friborg O, Martinussen M, Kaiser S, Øvergård KT, Rosenvinge JH. Comorbidity of personality disorders in anxiety disorders: a metaanalysis of 30 years of research. J Affect Disord 2013;145:143-155.

6. Starcevic V, Brakoulias V. New diagnostic perspectives on obsessivecompulsive personality disorder and its links with other conditions. Curr Opin Psychiatry 2014;27:62-67.

7. Gordon OM, Salkovskis PM, Oldfield VB, Carter N. The association between obsessive compulsive disorder and obsessive compulsive personality disorder: Prevalence and clinical presentation. Br J Clin Psychol 2013;52:300-315.

8. Beck JS. Complex cognitive therapy treatment for personality disorder patients. Bull Menninger Clin 1998;62:170-194.

9. Fricke S, Moritz S, Andresen B, Jacobsen D, Kloss M, Rufer M, et al. Do personality disorders predict negative treatment outcome in obsessive-compulsive disorders? A prospective 6-month follow-up study. Eur Psychiatry 2006;21:319-324.

10. Rufer M, Hand I, Alsleben H, Braatz A, Ortmann J, Katenkamp B, et al. Long-term course and outcome of obsessive-compulsive patients after cognitive-behavioral therapy in combination with either fluvoxamine or placebo: a 7-year follow-up of a randomized double-blind trial. Eur Arch Psychiatry Clin Neurosci 2005;255:121-128.

11. Lochner C, Fineberg NA, Zohar J, Van Ameringen M, Juven-Wetzler A, Altamura AC, et al. Comorbidity in obsessive-compulsive disorder (OCD): a report from the International College of Obsessive-Compulsive Spectrum Disorders (ICOCS). Compr Psychiatry 2014;55:15131519.

12. Hofmeijer-Sevink MK, Van Oppen P, Van Megen HJ, Batelaan NM, Cath DC, Van Der Wee NJA, et al. Clinical relevance of comorbidity in obsessive compulsive disorder: The Netherlands OCD Association study. J Affect Disord 2013;150:847-854.

13. Crino RD, Andrews G. Obsessive-compulsive disorder and axis I comorbidity. J Anxiety Disord 1996;10:37-46.

14. Beck AT, Beck JS. The Personality Belief Questionnaire. Unpublished Assessment Instrument. Pennsylvania: The Beck Institute for Cognitive Therapy and Research, Bala Cynwyd; 1991.

15. Turkcapar MH, Orsel S, Ugurlu M, Sargin E, Turhan M, Akkoyunlu S, et al. Reliability and validity of Turkish version of Personality Belief Questionnaire. J Clin Psychiatry 2007;10:177-191.

16. Butler AC, Beck AT, Cohen LH. The personality belief questionnaire- 
short form: Development and preliminary findings. Cognit Ther Res 2007;31:357-370.

17. Taymur I, Turkcapar MH, Orsel S, Sargin E. Validity and reliability of the Turkish cersion of the Personality Belief Questionnaire- Short Form (PBQ-STF) in the university students. J Clin Psychiatry 2011;14: 199-209.

18. Goodman WK, Price LH, Rasmussen SA, Mazure C, Fleischmann RL, Hill CL, et al. The Yale-Brown Obsessive Compulsive Scale: I. Development, use, and reliability. Arch Gen Psychiatry 1989;46:1006-1011.

19. Tek C, Uluğ B, Rezaki BG, Tanriverdi N, Mercan S, Demir B, et al. Yale-Brown Obsessive Compulsive Scale and US National Institute of Mental Health Global Obsessive Compulsive Scale in Turkish: reliability and validity. Acta Psychiatr Scand 1995;91:410-413.

20. Hisli N. Reliability and validity of Beck Depression Inventory among university students. Turk J Psychol 1989;7:3-13.

21. Beck AT, Epstein N, Brown G, Steer RA. An inventory for measuring clinical anxiety: Psychometric properties. J Consult Clin Psychol 1988;56:893-897.

22. Rees CS, Roberts LD, van Oppen P, Eikelenboom M, Hendriks AAJ, van Balkom AJLM, et al. Personality and symptom severity in Obsessive-Compulsive Disorder: the mediating role of depression. Pers Individ Dif 2014;71:92-97

23. Starcevic V, Brakoulias V. Current understanding of the relationships between obsessive-compulsive disorder and personality disturbance. Curr Opin Psychiatry 2017;30:50-55.

24. Torres AR, Moran P, Bebbington P, Brugha T, Bhugra D, Coid JW, et al. Obsessive-compulsive disorder and personality disorder: evidence from the British National Survey of Psychiatric Morbidity 2000. Soc Psychiatry Psychiatr Epidemiol 2006;41:862-867.

25. Melca IA, Yücel M, Mendlowicz MV, de Oliveira-Souza R, Fontenelle LF. The correlates of obsessive-compulsive, schizotypal, and borderline personality disorders in obsessive-compulsive disorder. J Anxiety Disord 2015;33:15-24.

26. Ansell EB, Pinto A, Edelen MO, Markowitz JC, Sanislow CA, Yen S, et al. The association of personality disorders with the prospective 7-year course of anxiety disorders. Psychol Med 2011;41:1019-1028.

27. Thiel N, Hertenstein E, Nissen C, Herbst N, Külz AK, Voderholzer U. The effect of personality disorders on treatment outcomes in patients with obsessive-compulsive disorders. J Pers Disord 2013;27:697-715.

28. Brakoulias V, Starcevic V, Belloch A, Brown C, Ferrao YA, Fontenelle LF, et al. Comorbidity, age of onset and suicidality in obsessive-compulsive disorder (OCD): An international collaboration. Compr Psychiatry 2017;76:79-86.

29. Olatunji BO, Rosenfield D, Tart CD, Cottraux J, Powers MB, Smits JA. Behavioral versus cognitive treatment of obsessive-compulsive disorder: an examination of outcome and mediators of change. J Consult Clin Psychol 2013;81:415-428.

30. Dèttore D, Pozza A, Coradeschi D. Does time-intensive ERP attenuate the negative impact of comorbid personality disorders on the outcome of treatment-resistant OCD? J Behav Ther Exp Psychiatry 2013;44:411417. 